\title{
Comparing the Success Rate and Side Effects of Endovenous Laser Ablation and Radiofrequency Ablation to Treat Varicose Veins in the Lower Limbs: A Randomized Clinical Trial
}

\author{
Arash Mohammadi Tofigh $^{1 *(\infty}$, Hamed Tahmasebi $^{2}{ }^{\circledR}$, Javad Zebarjadi ${ }^{3}$ \\ ${ }^{1}$ Laser Application in Medical Sciences Research Center, Shahid Beheshti University of Medical Sciences, Tehran, Iran \\ ${ }^{2}$ Assistant Professor, Department of General Surgery, School of Medicine, Shahid Beheshti University of Medical Sciences, \\ Tehran, Iran \\ ${ }^{3}$ Department of General Surgery, School of Medicine, Shahid Beheshti University of Medical Sciences, Tehran, Iran
}

\section{*Correspondence to Arash Mohammadi Tofigh, Laser Application in Medical Sciences Research Center, Shahid Beheshti University of Medical Sciences, Tehran, Iran. Email: arash_mtofigh@yahoo. com}

Published online December 30 2020

\begin{abstract}
Introduction: Varicosis is a condition affecting the superficial venous system of the lower limbs. Endovenous laser ablation (EVLA) is a minimally invasive method that uses different frequencies of laser for treatment. Radiofrequency (RF) has also been effectively used for vein ablation through thermal mechanisms. This study compares the success rate and side effects of EVLA and RF to treat varicose veins in the lower limbs within 12 months.

Methods: In this two-arm, parallel-group, randomized controlled, single-blind study, 1090 patients with lower limb varicosis proven by Doppler ultrasonography (DUS) were randomly placed into one of the two groups: EVLA and RF ablation. In the EVLA group, we used a 980-nm diode laser in a pulse mode and in the RF group, a 7-cm ClosureFast (Covidien, USA) catheter with an RF generator. We assessed pain during the operation and 8 and 24 hours after the surgery by the numerical rating scale (NRS). The patients were followed up on day 7 , then 3,6 , and 12 months postoperatively. The presence or absence of pain and then the severity of pain (according to NRS), the recurrence rate, complications, and the length of the procedure were compared.

Results: There was no significant difference between the two groups in the patients' characteristics, major adverse events and pain severity during the procedure and 8 and 24 hours after the surgery. According to NRS, the mean chronic pain severity in the EVLA group was $3.99 \pm 0.754$ versus $4.50 \pm 0.657$ in the RF group, and the difference was not significant. The length of operation and the recurrence rate 12 months after the procedure were not significantly different.

Conclusion: The results of our study further establish the efficacy of EVLT and RF ablation for lower limb varicosis treatment without any significant difference in the clinical outcomes and complications during a 12-months follow-up.

Keywords: Endovenous laser ablation; EVLA; Radiofrequency ablation; RF; Varicosis.
\end{abstract}

\section{Introduction}

Varicosis is a condition affecting the superficial venous system of thelower limbs. ${ }^{1}$ Its prevalence is estimated at $15 \%$ in men and $25 \%$ in women. ${ }^{2}$ One mechanism of the disease is reflux of blood through the saphenous vein due to the venous valve (duckbill valves) insufficiency, especially in the saphenofemoral junction, saphenopopliteal junction, or perforating veins. The malfunctioning valves can be in one or more of the mentioned places. ${ }^{3}$ In its early stages, varicosis can be asymptomatic, but as it progresses, pain, limb fatigue, edema, skin alterations, and venous ulcers develop..$^{4}$ If left untreated, severe complications such as thrombophlebitis and chronic venous insufficiency can occur. $^{3}$
The CEAP (Clinical-Etiology-AnatomyPathophysiology) classification is an internationally accepted standard for describing patients with chronic venous disorders, and it has been used for reporting clinical research findings in scientific journals (Figure 1). Based on CEAP, patients with varicose veins and secondary edema or changes in skin and subcutaneous tissue $(\mathrm{C} 2, \mathrm{C} 3, \mathrm{C} 4)$ are candidates to be treated with surgical or interventional methods, provided they do not have deep vein thrombosis (DVT) in Doppler ultrasonography (DUS) and have incompetent venous valves in the saphenous system. ${ }^{5}$

The greater saphenous vein (GSV) is the most commonly affected among symptomatic patients,

Please cite this article as follows: Mohammadi Tofigh A, Tahmasebi H, Zebarjadi J. Comparing the success rate and side effects of endovenous laser ablation and radiofrequency ablation to treat varicose veins in the lower limbs: a randomized clinical trial. J Lasers Med Sci. 2020;11(suppl 1):S43-S48. doi:10.34172/jlms.2020.S7. 


\begin{tabular}{|c|l|}
\hline C0 & No visible or palpable signs of venous disease \\
\hline C1 & Telangiectasias or reticular veins \\
\hline C2 & Varicose veins \\
\hline C2r & Recurrent varicose veins \\
\hline C3 & Edema \\
\hline C4 & Changes in skin and subcutaneous tissue secondary to chronic venous disease \\
\hline C4a & Pigmentation or eczema \\
\hline C4b & Lipodermatosclerosis or atrophie blanche \\
\hline C4c & Corona phlebectatica \\
\hline C5 & Healed \\
\hline C6 & Active venous ulcer \\
\hline C6r & Recurrent active venous ulcer \\
\hline & \\
\end{tabular}

Figure 1. Classification of Chronic Venous Disorders of the Lower Limbs Based on CEAP. Note. CEAP: Clinical manifestation, Etiology, Anatomic distribution, Pathophysiology,

followed by the lesser saphenous vein. ${ }^{6}$ Diagnosis of this condition is easily possible with color DUS, which shows the incompetent valves and reflux.

New methods for treating varicosis are endovenous laser ablation (EVLA), radiofrequency (RF) ablation, and sclerotherapy of GSV. ${ }^{7}$

EVLA is a minimally invasive method used in the treatment of lower limb varicosis with acceptable results. Laser frequencies that range from 810 to $1470 \mathrm{~nm}$ are used for this purpose. The laser causes clot formation in the blood vessels through its thermal effects, causing the vessel to occlude and block the blood flow. ${ }^{7}$ RF has also been effectively used for treatment through fiber and thermal mechanisms for vein ablation. RF ablation uses $\mathrm{RF}$ energy in 20-second bursts via a tiny catheter inserted into the vein. This energy targets the collagen in the vein wall. Targeting the vein wall causes the vein to shrink around the catheter as the surgeon treats the vein. The vein closes while the surgeon performs the treatment. ${ }^{8}$

Both EVLA and RF methods have already been introduced as suitable methods for the treatment of varicose veins and as safe and secure treatments in numerous articles and reference books. ${ }^{1,7-11}$ This study compares the success rate and side effects of EVLA and $\mathrm{RF}$ to treat varicose veins in the lower limbs within a 12-months follow-up.

\section{Materials and Methods}

All patients with lower limb varicosis who were referred to the Varice Clinic in Tehran, Iran from September 2018 to September 2019 underwent a lower limb venous DUS by an expert radiologist, and a report of a retrograde flow (reflux) for more than 0.5 seconds at the saphenofemoral joint (SFJ) was considered abnormal.

Based on CEAP classification, all patients in C2, C3, C4 stages who had 18-65 years old with SFJ reflux in DUS, were included in the study. Recurrent cases, impossibleto-follow-up patients, patients with pregnancy, active malignancy, acute or old DVT, an arterial occlusive disease with an ankle-brachial index of below 0.8 , known thrombophilia or high risk of pulmonary thromboembolism and history of inguinal surgery except for hernia were excluded. Finally, 1090 patients entered this two-arm parallel-group, randomized controlled, single-blind study. We performed simple randomization using the Rand function of Excel software (random number table). A mixture of numbers from 1 to 1090 was made, and patients entering the study got the next code from the table in order. If the number was below 545, we assigned the patient to the EVLA group, and If it was above 545 , to the RF ablation group. The patients were not aware of their group and were blinded. For each patient, we included only one limb in the study. For patients with bilateral disease, the leg with the most severe symptoms was included.

All patients underwent a complete physical examination and routine laboratory testing and the Aberdeen Varicose Vein Symptom Severity (AVVSS) scores were calculated and submitted.

After being transferred to the operating room (OR), the patients were put in the supine position, and the affected leg was temporarily raised above the level of the right ventricle. We used an elastic bandage to facilitate the drainage of the varicose veins. All Patients received mild intravascular sedation and were locally anesthetized under ultrasonographic guidance (Hitachi EUB-525F Ultrasound System, transducer 7.5-13 MHz linear/38 $\mathrm{mm}$, Hitachi EUP-L34T) with a solution containing 1 cc of $\mathrm{NaHCO}, 50 \mathrm{~mL}$ of $1 \%$ lidocaine, and $500 \mathrm{~mL}$ of normal saline. After injecting $1 \mathrm{~g}$ of prophylactic cefazolin intravenously, a 16-G access needle was inserted into the GSV near the knee toward the hip under ultrasonographic guidance and sterile conditions. Then, a 0.035-inch guidewire was introduced into the vein. The intravenous placement was confirmed with ultrasonography, and the introducer sheath was placed over the wire.

In the EVLA group, we used a long sheath, and a 600 -micron laser fiber was inserted into the sheath up to 2-5 mm out of the sheath tip. We used a $980-\mathrm{nm}$ diode laser in a pulse mode ( 1 second on and 0.5 seconds off or 100 milliseconds on/off), with $12 \mathrm{~W}$ power. The energy was delivered, and the fiber was carefully withdrawn down to the ablation starting point $(\mathrm{V}=2 \mathrm{~mm} / \mathrm{s})$ during the procedure.

In the RF group, we used an $11 \mathrm{~cm}$ sheath. A $7 \mathrm{~cm}$ ClosureFast (ClosureFast, Covidien/Medtronic, CA, USA) catheter was inserted into the sheath and pushed toward proximal and fixed 10-15 mm before the SFJ under sonographic guidance. The RF generator delivered RF energy to the ClosureFast catheter during the procedure. Every 20 seconds, we pulled $7 \mathrm{~cm}$ of the catheter back to perform overlapping segmental ablation and shrinkage of the vein. The controlled feedback mechanism monitored intravascular heat parameters in real-time to automatically regulate therapeutic power.

In patients with concomitant perforator insufficiency, these veins were surgically ligated through small incisions after laser therapy. After interventions, we used a 
compression bandage for all patients for 48 hours. The patients were instructed to continue using the bandage or a class II above the knee varicose stocking for 4-8 weeks. Acetaminophen $500 \mathrm{mg}$ was prescribed for all patients orally every six hours for three days after the procedure. We assessed pain during the operation and 8 and 24 hours after the surgery by the numerical rating scale (NRS).

In the NRS, patients are asked to circle the number between 0 and 10 that best fits their pain intensity. Zero usually represents 'no pain at all' whereas the upper limit represents 'the worst pain ever possible'. In contrast to other pain scales, only the numbers themselves are valuable answers, meaning only 11 possible answers. It thus allows only a less-subtle distinction of pain levels compared to other scales, where there is theoretically an unlimited number of possible answers. ${ }^{9}$

All patients were discharged on the same day of the operation.

We followed all patients on day 7, then 3, 6, and 12 months postoperatively. The patients underwent a physical examination by the same research team, and AVVSS scores were calculated and submitted on each follow-up visit. The presence or absence of pain and then the severity of pain (according to NRS) were questioned and recorded.

The same radiologist performed DUS on every visit to rule out recurrence.

The evaluating team and the radiologist were all unaware of the patients' group and were blinded.

We used SPSS 25 (IBM Inc., Armonk NY, USA) for data analysis. We used a chi-square test for nominal and ordinal variables and an independent t-test for the continuous variable.

\section{Results}

In total, 1090 patients were included in the study from January to September 2018. The patients were divided into the EVLA group ( $\mathrm{n}=530 ; 140$ males, 390 females) and the RF group ( $\mathrm{n}=560 ; 140$ males, 420 females). The mean age of the patients was $36.9 \pm 9.31$ years in the EVLT group and $37.26 \pm 9.54$ years in the RF group $(P=0.88)$. The left limb was affected in $67 \%$ of the EVLA group and $62 \%$ of the RF group $(P=0.750)$. Patient demographics are shown in Table 1.

According to NRS, the pain severity during the procedure in the EVLA group was $8.68 \pm 0.749$ versus $8.05 \pm 1.071$ in the RF group, and the difference was not significant $(P=0.066)$. Pain severity in 8 and 24 hours after the surgery was not significantly different between the two groups (Table 2).

Chronic pain (seventh day, 3, 6, and 12 months after the surgery) was present in $13.8 \%$ of the EVLA group and $15.8 \%$ of the RF group without a significant difference $(P$ $>0.05$; Table 3 ).

According to NRS, the mean chronic pain severity in the EVLA group was $3.99 \pm 0.754$ versus $4.50 \pm 0.657$
Table 1. Patients Demographics

\begin{tabular}{llccc}
\hline & & EVLA & RFA & P Value \\
\hline Age & & $36.9 \pm 9.31$ & $37.26 \pm 9.54$ & 0.888 \\
\multirow{2}{*}{ Sex } & Male & $140(26 \%)$ & $140(25 \%)$ & 0.864 \\
& Female & $390(74 \%)$ & $420(75 \%)$ & 0.864 \\
\multirow{2}{*}{ Limb } & Left & $355(67 \%)$ & $347(62 \%)$ & 0.750 \\
& Right & $175(33 \%)$ & $213(38 \%)$ & 0.750 \\
\hline
\end{tabular}

EVLA: endovenous laser ablation, RFA: radiofrequency ablation, AVVSS: aberdeen varicose vein symptom severity.

Table 2. Acute pain distribution according to NRS

\begin{tabular}{|c|c|c|c|c|}
\hline & & Pain Score & Standard Deviation & $P$ Value \\
\hline \multirow{2}{*}{ Pain during procedure } & EVLA & 8.68 & 0.749 & \multirow{2}{*}{0.066} \\
\hline & RFA & 8.05 & 1.071 & \\
\hline \multirow{2}{*}{ Pain at 8 hours } & EVLA & 7.97 & 1.079 & \multirow{2}{*}{0.085} \\
\hline & RFA & 7.57 & 1.076 & \\
\hline \multirow{2}{*}{ Pain at 24 hours } & EVLA & 5.58 & 1.017 & \multirow{2}{*}{0.0754} \\
\hline & RFA & 4.67 & 1.044 & \\
\hline
\end{tabular}

EVLA: endovenous laser ablation, RFA: radiofrequency ablation.

Table 3. Chronic Pain Distribution

\begin{tabular}{lcccc}
\hline & & EVLA & RFA & P Value \\
\hline \multirow{2}{*}{ Chronic pain } & Yes & $73(13.8 \%)$ & $88(15.8 \%)$ & \multirow{2}{*}{0.9} \\
& No & $457(86.2 \%)$ & $472(84.2 \%)$ & \\
Chronic pain score (NRS) & $3.99 \pm 0.754$ & $4.50 \pm 0.657$ & 0.78 \\
\hline
\end{tabular}

EVLA: endovenous laser ablation, RFA: radiofrequency ablation, NRS: numerical rating scale.

in the RF group, and the difference was not significant $(P>0.05$; Table 3$)$.

The length of the operation was $44.5 \pm 11.2$ minutes in the EVLA group versus $40.3 \pm 10.6$ minutes in the RF group that was not significantly different $(P=0.14$; Table 4).

At 12 months, the recurrence rate of the EVLA and RF groups was 6.7 and $5.7 \%$ respectively $(P=0.08$; Table 4). The prevalence of different side effects and scores is presented in Table 5, which were not significantly different in both groups on follow-up.

No significant difference was observed between the two treatment groups in major adverse events like DVT, dysesthesia and pigmentation (Table 5).

Despite an initial increase in the first hours, the AVVSS score significantly declined in both groups after treatment; still, it was not significantly different between the groups at 12 months of follow-up. We observed a maximum decline in symptoms between one week and three months postoperatively in both groups (Table 6). Patient satisfaction was similar in both groups at 3,6, and 12 months of follow-up (Table 5). 
Table 4. Clinical Results

\begin{tabular}{lcccc}
\hline & EVLA & RFA & P Value \\
\hline Length of operation (min) & $44.5 \pm 11.2$ & $40.3 \pm 10.6$ & 0.066 \\
Recurrence rate & & $6.7 \%$ & $5.7 \%$ & 0.08 \\
& At 3 months & $478(92 \%)$ & $520(93 \%)$ & 0.999 \\
$\begin{array}{l}\text { Patient satisfaction } \\
\text { In follow-up }\end{array}$ & At 6 months & $493(93 \%)$ & $526(94 \%)$ & 0.890 \\
& At 12 months & $493(93 \%)$ & $526(94 \%)$ & 0.890 \\
\hline
\end{tabular}

EVLA: endovenous laser ablation, RFA: radiofrequency ablation.

Table 5. Prevalence of Side Effects on Follow-up

\begin{tabular}{|c|c|c|c|c|}
\hline Symptom & Follow-up & $\begin{array}{c}\text { EVLA } \\
\text { No }(\%)\end{array}$ & $\begin{array}{c}\text { RFA } \\
\text { No }(\%)\end{array}$ & $P$ value \\
\hline \multirow{3}{*}{ Bruising } & At 3 months & $382(70 \%)$ & $268(45 \%)$ & 0.035 \\
\hline & At 6 months & 208 (35\%) & $131(22 \%)$ & 0.05 \\
\hline & At 12 months & $60(10 \%)$ & $54(9 \%)$ & 0.89 \\
\hline \multirow{3}{*}{ Pigmentation } & At 3 months & 137 (23\%) & $178(30 \%)$ & 0.04 \\
\hline & At 6 months & 89 (15\%) & 89 (15\%) & 1 \\
\hline & At 12 months & $36(6 \%)$ & $30(5 \%)$ & 0.98 \\
\hline \multirow{3}{*}{ Dysesthesia } & At 3 months & 89 (15\%) & $119(20 \%)$ & 0.75 \\
\hline & At 6 months & $48(8 \%)$ & $60(10 \%)$ & 0.89 \\
\hline & At 12 months & $24(4 \%)$ & $30(5 \%)$ & 0.99 \\
\hline \multirow{3}{*}{ DVT } & At 3 months & $1(0.18 \%)$ & $2(0.35 \%)$ & 0.06 \\
\hline & At 6 months & 0 & 0 & 1 \\
\hline & At 12 months & 0 & 0 & 1 \\
\hline
\end{tabular}

EVLA: endovenous laser ablation, RFA: radiofrequency ablation.

Table 6. AVVSS Score Measured at Each Follow-up

\begin{tabular}{lccc}
\hline Visit & $\begin{array}{c}\text { EVLA } \\
\text { Mean } \pm \text { SD }\end{array}$ & $\begin{array}{c}\text { RFA } \\
\text { Mean } \pm \text { SD }\end{array}$ & P Value \\
\hline Screening & $4.7 \pm 3.1$ & $4.9 \pm 2.8$ & 0.690 \\
8 hours & $4.7 \pm 1.6$ & $5.0 \pm 2.5$ & 0.05 \\
24 hours & $4.2 \pm 1.5$ & $4.3 \pm 2.0$ & 0.89 \\
3 months & $4.0 \pm 1.8$ & $4.1 \pm 1.2$ & 0.90 \\
6 months & $2.7 \pm 2.2$ & $3.1 \pm 1.5$ & 0.780 \\
12 months & $1.9 \pm 2.0$ & $1.8 \pm 2.0$ & 0.890 \\
\hline
\end{tabular}

AVVSS: The Aberdeen Varicose Vein Symptom Severity, EVLA: endovenous laser ablation, RFA: radiofrequency ablation.

\section{Discussion}

Varicosis in lower limbs is defined as distended and tortuous superficial veins that characterize venous insufficiency due to venous valve malfunction and reduced elasticity of vessel walls. ${ }^{1}$ It is reported that $3.6 \%$ of the general population suffer from varicose veins. The condition affects women twice as much as men, and its prevalence increases to $15 \%$ in men and $25 \%$ in women aged 40 and over. ${ }^{2}$ The basis for the lower prevalence in males has not been clarified. Still, such variations have been attributed to differences in the variability of study populations, including age, gender, race, disease definition, and measurement. ${ }^{4}$ It is more common in people who stand for many hours for work reasons, such as rug weavers and nurses. ${ }^{6,7}$ This makes varicosis a common complaint among the working-class population, who seek faster relief of symptoms and quicker return to work.

Standard treatment strategies for varicosis include stripping, ligation of the SFJ, and phlebectomy. ${ }^{6,9}$ Conventional surgical treatments carry a $33 \%$ chance of recurrence and are accompanied by side effects such as scars, infections, and bleeding. ${ }^{12,13}$ Surgeons have turned to minimally invasive procedures to lower the rate of such side effects. Some of these procedures are EVLA, RF ablation (RFA), and sclerotherapy under ultrasonographic guidance.

EVLA and RFA are both effective intravascular methods, but the mechanisms of their effects are different. Both methods can be performed on an outpatient basis with local anesthesia, and they are faster and produce less pain and smaller scars compared to conventional surgery. ${ }^{8}$

Navaro et al first described the EVLA procedure in $2001 .^{13}$ They reported the successful results of EVLT on 40 patients with four months of follow-up. Initially, this procedure was only used for GSV insufficiencies, but later reports confirmed its effectiveness in the lesser saphenous vein and perforating veins. ${ }^{14}$ At 6 months, the success rate for EVLA has been reported to be between $95 \%$ and $100 \% .{ }^{15}$ Endovenous laser devices have frequencies that range from 810 to $1470 \mathrm{~nm}$. The laser energy is delivered to the venous wall via fiber and causes clot formation in the blood vessel, causing the vessel to occlude and block the blood flow. ${ }^{16,17}$

RF has also been effectively used for treatment through fiber and thermal mechanisms for vein ablation. RF ablation uses RF energy in 20-second bursts via a catheter inserted into the vein. ${ }^{11}$ The procedure utilizes overlapping segmental RF ablation to deliver uniform heat to close the vein. This energy targets the collagen in the vein wall. Targeting the vein wall causes the vein to shrink around the catheter as the surgeon treats the vein. The vein closes while the surgeon performs the treatment. Once sealed, blood is directed to nearby healthy veins. ${ }^{18,19}$

Our study was a randomized controlled trial comparing EVLT and RF ablation.

The primary clinical results of the procedures did not differ between the two groups; $90 \%$ of the patients in both treatment groups expressed symptom relief. Intraand post-procedural pain, tenderness, and ecchymosis were slightly prevalent in the laser-treated limbs but not significant. All scores referable to pain and tenderness were statistically similar between the two groups at 8 hours, 24 hours, 1 week, 6, and 12 months. Minor complications were more prevalent in the EVLA group $(P=0.0210)$; there were no major complications. We also calculated the AVVSS score for all patients on each follow-up visit and compared the results. ${ }^{20}$ In the RF 
versus vein stripping trial reported by Lurie et al, ${ }^{21}$ mean AVVSS was reduced from a pretreatment score of 4.8 to 2.5 at 3 weeks after the treatment. This is similar to the extent of reduction seen in the present trial, in which the AVVSS of 4.9 at the baseline decreased on each follow-up visit, ending at 1.8 in the RF group and from 4.7 to 1.9 in the EVLA group at 12 months after treatment. The results suggest that patients treated with RF ablation take slightly longer to show improvement beyond pretreatment levels.

In the present trial comparing ClosureFAST RF catheter treatment versus 980-nm EVLA, we observed excellent correction of parameters linked to venous reflux, especially venous hypertension secondary to GSV incompetence irrespective of the device used for the treatment. All indices measuring post-inflammatory sequelae demonstrated no difference favoring RF ablation versus EVLA.

It is reported that patients better tolerate the RF procedure because controlled heating avoids the vein perforations often seen with EVLA. Laser wavelengths based on the affinity of hemoglobin for infrared light have effectively destroyed incompetent veins at the expense of causing perivenous inflammation. ${ }^{19}$ To overcome the problem of venous perforation, EVLA technology continues moving further toward the development of longer wavelengths targeting the last peak of water absorption. The idea of hemoglobin absorption is bypassed, allowing more robust absorption of laser photons by interstitial water in the vein wall. By targeting the vein wall, EVLA may improve its postoperative recovery profile. As the experience of working with Lasers increases, such side effects are reduced. ${ }^{22}$

Interestingly, targeting the vein wall exclusively has always been the goal of RF ablation. This has been true from the first device operating at $85^{\circ} \mathrm{C}$ to our catheters operating at $120^{\circ} \mathrm{C}^{23}$ In the original bipolar RF ablation procedure, the vein wall served as a resistive element for the transfer of energy from anode to cathode; but in the contemporary ClosureFAST device, the vein wall is the direct recipient of conducted heat from a $7-\mathrm{cm}$-long heating element, and the temperature is kept stable at $120^{\circ} \mathrm{C}$ during a 20 -second treatment cycle. ${ }^{24}$

Interestingly, RF ablation seems to have benefits with a lower risk of overall complication than EVLA. ${ }^{25}$ However, in our study, both procedures were well-tolerated without a significant difference, and our study failed to show any significant difference between the two groups regarding patient satisfaction.

\section{Conclusion}

The results of this study indicate the efficacy of EVLT and $\mathrm{RF}$ ablation for lower limb varicosis treatment. They also prove that the two methods are not significantly different in length of procedure, complications, and pain. The cosmetic outcome of both methods is acceptable. The one-year follow-up results indicate no difference in the recurrence rate and pain between the two groups. These findings will have to be further validated in larger studies before concrete recommendations can be made.

\section{Ethics Considerations}

The study protocol was approved by the Ethics committee of the national institute for health research (code: IR.TUMS.NIHR.REC.1397.016) and was registered in the Iranian Registry of Clinical Trials (identifier: IRCT20200404046936N4). All patients were enrolled in the study after they provided informed consent.

\section{Conflict of Interests}

Authors declare that they have no conflict of interest.

\section{Consent to participate}

All Patients were enrolled in the study after they provided informed consent.

\section{Funding}

This work was supported by the Vice-Chancellor's Office in Research Affaires of Shahid Beheshti University of Medical Sciences.

\section{References}

1. Bartholomew JR, King T, Sahgal A, Vidimos AT. Varicose veins: newer, better treatments available. Cleve Clin J Med. 2005;72(4):312-8. doi: 10.3949/ccjm.72.4.312.

2. Robertson L, Evans C, Fowkes FG. Epidemiology of chronic venous disease. Phlebology. 2008;23(3):103-111. doi: 10.1258/phleb.2007.007061.

3. Labas P, Cambal M. Profuse bleeding in patients with chronic venous insufficiency. Int Angiol. 2007;26(1):64-66.

4. Marchiori A, Mosena L, Prandoni P. Superficial vein thrombosis: risk factors, diagnosis, and treatment. Semin Thromb Hemost. 2006;32(7):737-743. doi: 10.1055/s-2006951459.

5. Lurie F, Passman M, Meisner M, Dalsing M, Masuda E, Welch $\mathrm{H}$, et al. The 2020 update of the CEAP classification system and reporting standards. J Vasc Surg Venous Lymphat Disord. 2020;8(3):342-52. doi: 10.1016/j.jvsv.2019.12.075.

6. Dwerryhouse S, Davies B, Harradine K, Earnshaw JJ. Stripping the long saphenous vein reduces the rate of reoperation for recurrent varicose veins: five-year results of a randomized trial. J Vasc Surg. 1999;29(4):589-592. doi: 10.1016/s0741-5214(99)70302-2.

7. Teo TK, Tay KH, Lin SE, Tan SG, Lo RH, Taneja M, et al. Endovenous laser therapy in the treatment of lower-limb venous ulcers. J Vasc Interv Radiol. 2010;21(5):657-662. doi: 10.1016/j.jvir.2010.01.029.

8. Luebke T, Brunkwall J. Systematic review and meta-analysis of endovenous radiofrequency obliteration, endovenous laser therapy, and foam sclerotherapy for primary varicosis. J Cardiovasc Surg (Torino). 2008;49(2):213-33.

9. Enzler MA, van den Bos RR. A new gold standard for varicose vein treatment? Eur J Vasc Endovasc Surg. 2010;39(1):97-98. doi: 10.1016/j.ejvs.2009.09.008.

10. Navarro L, Min RJ, Bone C. Endovenous laser: a new 
minimally invasive method of treatment for varicose veins-preliminary observations using an $810 \mathrm{~nm}$ diode laser. Dermatol Surg. 2001;27(2):117-122. doi: 10.1046/j.1524-4725.2001.00134.x.

11. Luebke T, Gawenda M, Heckenkamp J, Brunkwall J. Metaanalysis of endovenous radiofrequency obliteration of the great saphenous vein in primary varicosis. J Endovasc Ther. 2008;15(2):213-23. doi: 10.1583/07-2287.1.

12. Durkin MT, Turton EP, Scott DJ, Berridge DC. A prospective randomised trial of PIN versus conventional stripping in varicose vein surgery. Ann R Coll Surg Engl. 1999;81(3):171-174.

13. Van Rij AM, Jiang P, Solomon C, Christie RA, Hill GB. Recurrence after varicose vein surgery: a prospective longterm clinical study with duplex ultrasound scanning and air plethysmography. J Vasc Surg. 2003;38(5):935-943. doi: 10.1016/s0741-5214(03)00601-3.

14. Proebstle TM, Gul D, Kargl A, Knop J. Endovenous laser treatment of the lesser saphenous vein with a $940-\mathrm{nm}$ diode laser: early results. Dermatol Surg. 2003; 29(4):357-361

15. Min RJ, Zimmet SE, Isaacs MN, Forrestal MD. Endovenous laser treatment of the incompetent greater saphenous vein. J Vasc Interv Radiol. 2001;12(10):1167-1171. doi: 10.1016/ s1051-0443(07)61674-1.

16. Proebstle TM, Moehler T, Gul D, Herdemann S. Endovenous treatment of the great saphenous vein using a $1,320 \mathrm{~nm}$ Nd:YAG laser causes fewer side effects than using a $940 \mathrm{~nm}$ diode laser. Dermatol Surg. 2005;31(12):16781684. doi: 10.2310/6350.2005.31308.

17. Corcos L, Dini S, De Anna D, Marangoni O, Ferlaino E, Procacci $\mathrm{T}$, et al. The immediate effects of endovenous diode $808-\mathrm{nm}$ laser in the greater saphenous vein: morphologic study and clinical implications. J Vasc Surg. 2005;41(6):1018-1025. doi: 10.1016/j.jvs.2005.03.002.

18. Rasmussen LH, Bjoern L, Lawaetz M, Blemings A, Lawaetz B, Eklof B. Randomized trial comparing endovenous laser ablation of the great saphenous vein with high ligation and stripping in patients with varicose veins: short-term results. J Vasc Surg. 2007;46(2):308-315. doi: 10.1016/j. jvs.2007.03.053.

19. Almeida JI, Kaufman J, Göckeritz O, Chopra P, Evans MT, Hoheim DF, et al. Radiofrequency endovenous ClosureFAST versus laser ablation for the treatment of great saphenous reflux: a multicenter, single-blinded, randomized study (RECOVERY study). J Vasc Interv Radiol. 2009;20(6):752-9. doi: 10.1016/j.jvir.2009.03.008.

20. Rutherford RB, Padberg FT Jr, Comerota AJ, Kistner RL, Meissner MH, Moneta GL. Venous severity scoring: an adjunct to venous outcome assessment. J Vasc Surg. 2000;31(6):1307-1312. doi: 10.1067/mva.2000.107094.

21. Lurie F, Creton D, Eklof B, Kabnick LS, Kistner RL, Pichot $\mathrm{O}$, et al. Prospective randomised study of endovenous radiofrequency obliteration (closure) versus ligation and vein stripping (EVOLVeS): two-year follow-up. Eur J Vasc Endovasc Surg. 2005;29(1):67-73. doi: 10.1016/j. ejvs.2004.09.019.

22. Mozafar M, Atqiaee K, Haghighatkhah H, Sanei Taheri M, Tabatabaey A, Lotfollahzadeh S. Endovenous laser ablation of the great saphenous vein versus high ligation: long-term results. Lasers Med Sci. 2014;29(2):765-71. doi: 10.1007/ s10103-013-1389-z.

23. Tofigh A M, Karvandi M, Coscas R. Current incidence of peripheral arterial embolism and role of echocardiography. Asian Cardiovasc Thorac Ann. 2008;16(6):439-443. doi: 10.1177/021849230801600602.

24. Bozoglan O, Mese B, Eroglu E, Ekerbiçer HC, Yasim A. Comparison of endovenous laser and radiofrequency ablation in treating varices in the same patient. $J$ Lasers Med Sci. 2017;8(1):13-16. doi: 10.15171/jlms.2017.03.

25. He G, Zheng C, Yu MA, Zhang H. Comparison of ultrasound-guided endovenous laser ablation and radiofrequency for the varicose veins treatment: An updated meta-analysis. Int J Surg. 2017;39:267-275. doi: 10.1016/j.ijsu.2017.01.080. 